
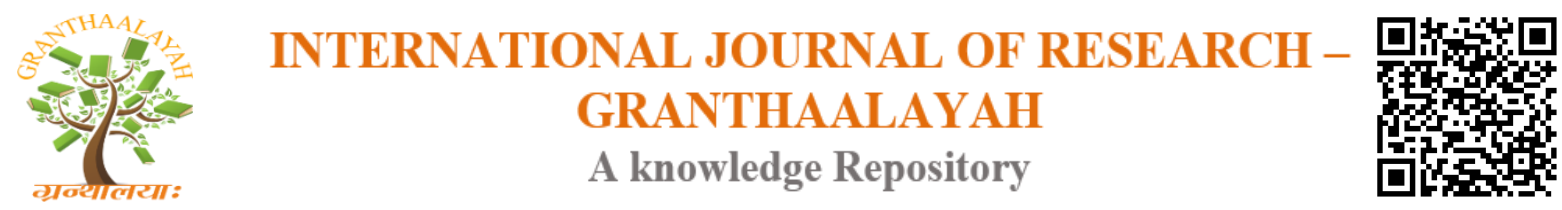

Science

\title{
PRINCIPLES OF DATAFLOW COMMUTATION OF OPTICAL NETWORKS
}

\author{
V.A.Maharramov ${ }^{* 1}$, M.G.Hasanov ${ }^{2}$ \\ ${ }^{* 1,2}$ Azerbaijan Technical University, Baku, Azerbaijan
}

\begin{abstract}
The principle of constructing a multi-channel and multifunctional optical commutator is analyzed. It is shown that, the proposed principle of an optical commutator extends the functional capabilities of optical network.
\end{abstract}

Keywords: Multi-Channel and Multifunctional Optical Commutators; Dataflow and Fiber-Optic Networks.

Cite This Article: V.A.Maharramov, and M.G.Hasanov. (2017). "PRINCIPLES OF DATAFLOW COMMUTATION OF OPTICAL NETWORKS." International Journal of Research - Granthaalayah, 5(12), 348-356. 10.29121/granthaalayah.v5.i12.2017.512.

\section{Introduction}

As it is known, the main advantage of fiber-optic networks is their practically unlimited capacity. The practical value of this property lies in the possibility of a frequent increase in information transfer speed via fiber optic communication channels on a global scale. And this makes the research in the field of optical networks very relevant and perspective.

Therefore, the world of telecommunications and data transmission is mainly connected with the increase in the number of Internet users, as well as with growing interaction of international operators and the increase in the amount of the transferred information. In addition to the important task of improving the parameters and constructing fiber-optic cables, the task of creating reliable commutators for optical signals is equally acute [1,2], without which it is impossible to construct multifunctional and branched optical networks.

The proposed work analyzes one of the possible principles for creating multi-channel and multifunctional optical commutators (or cross-commutator) for dataflow. Since ray propagation trajectory in optical devices has a reciprocal nature, therefore, while the operation principle of the proposed optical commutator is being described in one operating mode, it is realized that, the reverse mode of this optical commutator is easily carried out by turning all flows in the opposite direction. In other words, optical systems are reversible and it suffices to describe the ray course in one direction. Therefore, all signal transmission lines can be bidirectional and signals in both 
directions are transmitted by light flux having different wavelengths in the commutators considered below.

One of the recent times perspective technological directions is the technology of a fully optical network (All-Optical Networks), where all operations on the transformation and formation of user information are carried out without transformation of the signal from optical form to the electrical one and vice versa [3]. Eliminating dataflow transformation into an electrical form makes it possible to significantly reduce the cost of the network, but the possibilities of optical technologies are still insufficient to create completely optical networks of the required scale. Therefore, practical application of such networks is limited by subnetworks, between which the electrical regeneration of the signal is still performed. However, some work in this direction is being intensively conducted. Currently, development of a number of active and passive quantum and optical elements and devices with highly stable parameters are being carried out. Among the passive elements, the most important are the optical commutators.

\section{A Commutator Based on Semi-Reflecting Mirrors}

Recall that, commutators are one of the most important nodes of information transmission systems, constructed on the basis of hierarchical structures. Without them, it is almost impossible to automatically control the shift of optical dataflow and monitor the branched network.

In the generalized sense a commutator is usually called a device allowing to transmit instantaneous initial signal with minimal distortion entering one of several inputs, or one of several outputs in accordance with the specified algorithm switching. Accordingly, optical commutators are the ones commuting signals represented in the form of optical radiation.

A huge variety of devices applied in technology represents a great interest of static commutator performing the function of optical signals commutation $[4,5]$. Commutators of this type have a low rate of constructing a signal transmission route, since with each route change it is necessary to mechanically change the position of one or several mirrors in the path of light flux propagation. However, in the absence of route changes, such commutators have the maximum data transmission rate (the signal is transmitted at velocity of light) independent on the data format and the wavelength of the light flux. On the other hand, commutators based on traveling mirrors have a low cost.

As mentioned in [4], the feature of static commutators is that the connections are set by the control unit for a relatively long period of time and can change, for instance, related with the connection to the system of previously unused devices, redistribution of traffic depending on the time of a day, with the failure of some external fiber-optic communication line and during reserve line switching with turnover time not more than $50 \mathrm{~ms}$.

As an example, the principle of constructing and functioning the commutator, consisting of 3 inputs and 3 outputs shown in fig. 1, is considered. Note that, it is possible to construct a commutator consisting of inputs $M$ and outputs $M$ on the base of the following principle of construction and operation. At the same time, at output $M x M$ of the commutator combinations 
$\left(M^{2}-1\right) \cdot M$ of input streams can be created (it is possible to create 24 combinations at the output), as well as to change the optical flows in the reverse direction, as mentioned above.

As it is seen from fig. 2, the commutator transmits optical signals from three input ports Bx1 Bx 3 to three output ports: output 1 - output 3. In this simplified example, the commutator contains the matrix of 9 traveling semi-reflecting mirrors located at the intersections of three rows and three columns. The step of placing semi-reflecting mirrors in the matrix is indicated through $d L$ and it depends on the overall dimensions of the mirrors' actuators (linear drive of reciprocal motion). Each semi-reflecting mirror can be in two stable states. One of the states of semi-reflecting mirrors is initial or passive (flush), in which the semi-reflecting mirror does not intersect the path of optical flow and is indicated by broken lines in the commutator diagram. The second state of semi-reflecting mirrors is an elevated one or active (vertical), in which a semi-reflecting mirror intersects the path of the optical flow, and divides it into two mutually perpendicular flows with equal power (intensity). Transition to the active state of semi-reflecting mirrors is carried out via linear displacements drive. In the commutator diagram, the active state is shown in the form of solid lines.

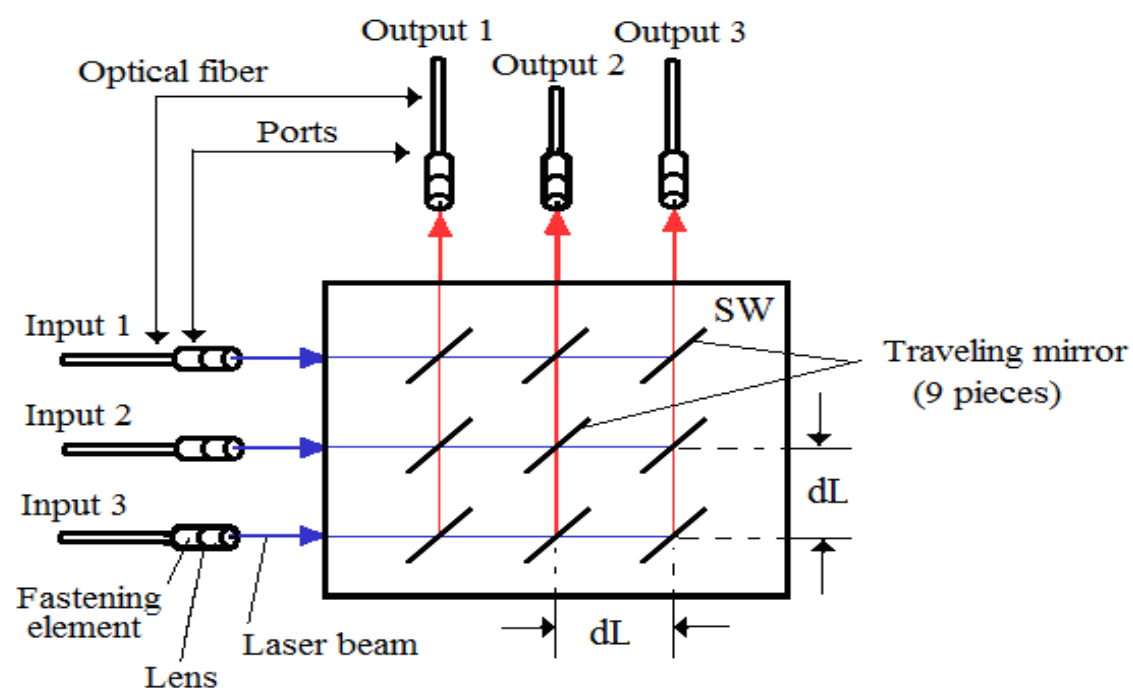

Figure 1: Diagram of optical commutator SW based on traveling mirrors

It is obvious that, the transition velocity of a semi-reflecting mirror from one state to another one determines the optical commutator speed. As it is seen from fig. 1 (where incidentally, the state of all semi-reflecting mirrors is shown as active), each port can additionally contain the elements for attaching the optical fiber to the commutator body and lens, which corrects the spatial divergence of the laser beam during its propagation.

\section{Implementation Options}

It should be noted that, the optical commutator sections shown in fig. 1, on a row or column, will have the same appearance. Therefore, one of the possible options for the appearance of an optical commutator is shown in fig. 2. 


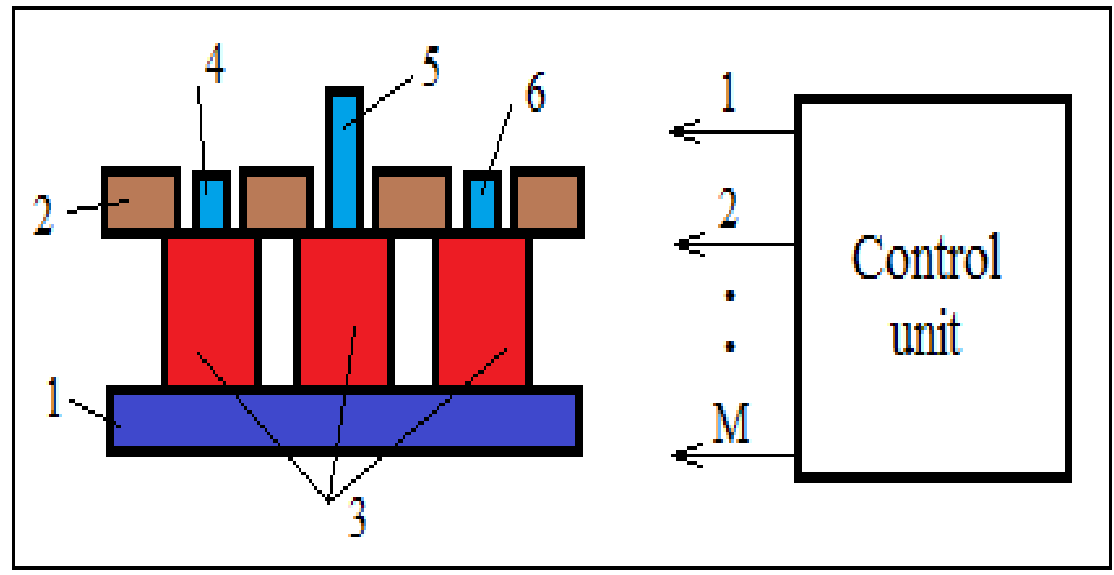

Figure 2: Commutator section: 1-base, 2-top cover, 3 linear movement drive, 4 - the first, 5 - the second, 6 - third semi-reflecting mirror

As it is seen from fig. 2, the commutator consists of a control unit, a base-1, an upper cover-2, linear drives of reciprocal motion-3, semi-reflecting mirrors-4, 5, 6. All semi-reflecting mirrors are rigidly fixed on the movable part of the drive.

The working surface of all semi-reflecting mirrors 4, 5 and 6 is covered with a layer of the required reflection coefficient and optical flow transmission. In fig. 2, semi-reflecting mirrors 4 and 6 are in the passive (flush), and the semi-reflecting mirror 5 is in the active (vertical) state. The operating position of the mirrors is set by the control voltage of the control unit, which is determined by the state of the network.

As it is seen from fig. 2, the optical commutator has sufficiently simple construction and its dimensions are determined by the size of linear movement drive.

While the commutator is being implemented as a drive, electromagnetic (figure 3), systems with flat spring-loaded plates (fig. 4) [6], packet and differential [7] piezo-motors (fig.5), piezoelectric deflectors, reversing drives [8-10] and other micro electromechanical and piezoceramic drives $[11,12]$.

The minimum dimension of miniature linear piezoelectric drives [13] is $1.55 \times 1.55 \times 6 \mathrm{~mm}$, the working stroke is $33 \mathrm{~mm}$ with a resolution of $50 \mathrm{~nm}$ and movement speed is up to $80 \mathrm{~mm} / \mathrm{s}$. The characteristic of the current linear displacement achieves micron and submicron accuracy [14]. It is possible to create miniature and full optical commutators of high accuracy and speed of commutating dataflow by using these linear drives.

Each linear actuator under the action of signals from the control unit (shown in figure 2 as a square) can be in a passive (flush) or active (vertical) position.

As it is seen from fig. 3 and fig. 4, a semi-reflecting mirror with a passive position can skip the optical beam (fig. 3, a and fig. 4, a), or divert the beam at an angle by dividing it into two equal flows (see fig. 3, b and fig. 4, b). 

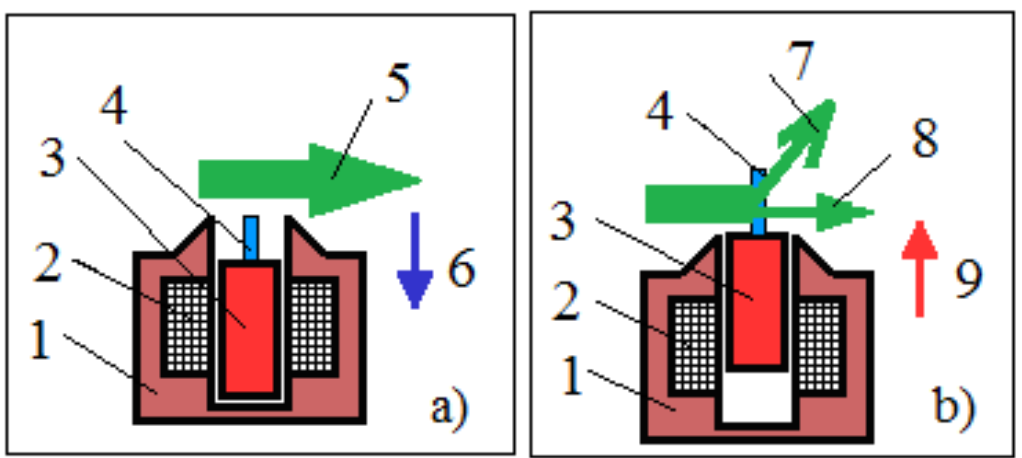

Figure 3: Electromagnetic drive of reciprocal motion

1-drive base, 2-solenoid, 3-armature (anchor), 4-semi-reflecting mirror, 5-optical beam of direct path, 6- armature (anchor) movement direction (drowning semi-reflecting mirror) down, 7deflected beam, 8-ray traversed through a semi-reflecting mirror, 9- armature (anchor) movement direction (raising the semi-reflecting mirror) upward.
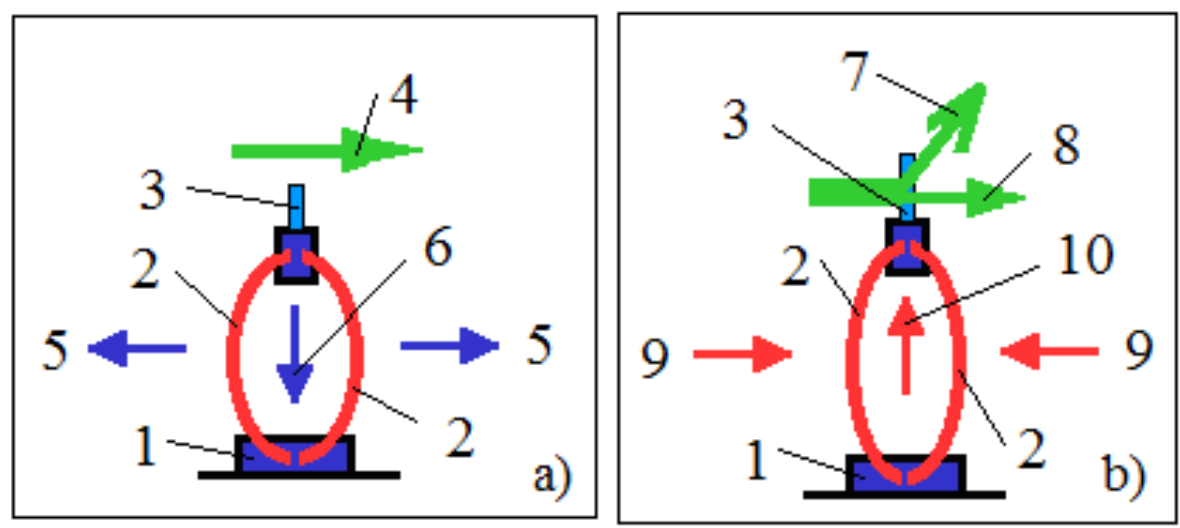

Figure 4: Kinematics of the system with flat spring-loaded plates

1-base, 2-spring-loaded plates, 3-semi-reflecting mirror, 4-optical beam of direct path, 5-plate drawing force, 6-direction of system height decrease (semi-reflecting mirror drowning) down, 7deflected beam, 8 - beam passed through a semi-reflecting mirror, 9- compression force of plates, 10-direction of increasing the height of the system (the pad of a semi-reflecting mirror) upward A distinctive feature of the system with flat spring-loaded plates (fig. 4) is that, when the operating voltage is removed thanks to spring-loaded drive [15], the system returns to the initial state. In the system, both an electromagnetic and a piezoelectric drive of reciprocal motion can be used as a drive.

Piezo-motors of linear displacements operate respectively in sub micrometer and micrometer, second and minute ranges. Their frequency range is limited to the area up to the first electromechanical resonance.

Batch construction [7] is built on the base of packet type actuators. They can consist of disks, rings, cylinders and so on. Fig.5a presents the simplest version of such an engine construction. The piezo-package 2 consists of piezodiscs and power transmitting pads. It is installed in body 1. 
Mechanical contact of rod 3 and body 1 are carried out through steel centering balls 5 . The plate spring 4 serves to provide a preliminary compression of the package. Semi-reflecting mirror 6 is rigidly fixed on working element 3 . Such an engine serves for linear micro-displacements.
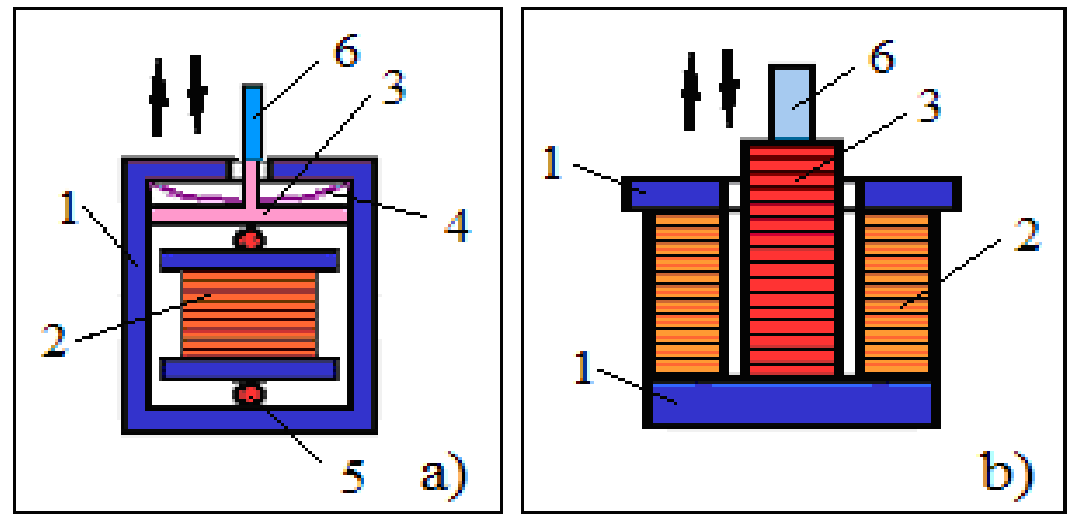

Figure 5: Batch and differential piezoelectric motor of translation

1-body, 2-piezo-packet, 3-working element (rod), 4-spring, 5-steel ball, 6-semi-reflecting mirror The frequency range of a packet piezo-electric motor is limited by its own electromechanical resonance, the value of which depends on the mass of the object being moved, and the movement range from 2 to $50 \mathrm{mkm}$ depends on the number of elements and the control voltage.

Fig.5, b presents a differential construction for batch-type elements, which is used when it is necessary to compensate the temperature error (thermal expansion of the working element) and increase the displacement range without significantly increasing the amount of control voltage and actuator dimensions.

Such a construction can be built both on batch type and bimorph elements [7]. Working element 3 consists of piezo-electric elements prepared in the form of washers (disks), however, element 2 consists of rings. Both elements are fixed to the movable base 1 . Free butt-end of the element 3 carries out working movements, on which semi-reflecting mirrors 6 are rigidly fixed. Control voltage is transmitted simultaneously to both elements, so that, element 2 expands, and 3 is compressed.

\section{Principle of the Commutator Operation}

Below some samples of the principle of optical commutators operation are considered. Each mirror can be in a passive (flush) or active (vertical) state. Later on, the active mirrors are shown in the form of thick solid lines, and the passive ones in the form of thin lines. As mentioned above, the working surface of all semi-reflecting mirrors is covered with a layer of $50 \%$ reflection coefficient and $50 \%$ optical flow transmission.

In the sample presented in fig.6, a, all light rays are divided into flows with the required power. Light beam $\lambda_{1}$ from input 1 port is reflected by the semi-reflecting mirror and enters the port out 2. Light beam $\lambda_{2}$ from input 2 port is transmitted to input 3 port. Finally, light beam from input 3 port enters input 1 port. 
In the sample presented in fig. $6, \mathrm{~b}$ all light rays are anologically divided into flows with the required power. Flows $\lambda_{1}, \lambda_{2}$ and $\lambda_{3}$ from inputs 1,2 and 3 , also pass an analogous path according to figure $6 \mathrm{a}$ and in addition, in contrast to fig. 6, a, all flows $\lambda_{1}, \lambda_{2}$ and $\lambda_{3}$ simultaneously are accordingly transmitted to inputs 4,5 and 6 in the form of $\lambda_{1}, \lambda_{2}$ and $\lambda_{3}$.

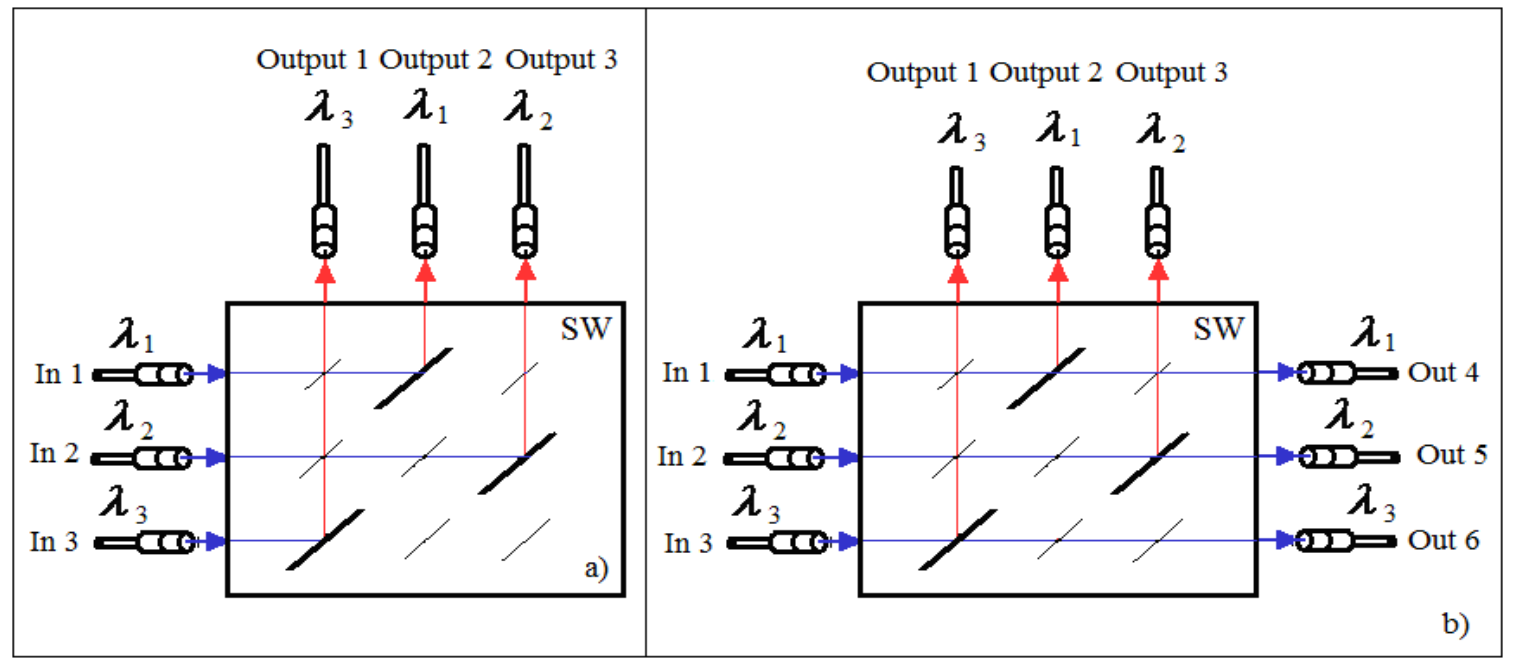

Figure 6: Principle of optical repeater commutator operation

In the sample presented in fig.7, a all light rays are divided into flows with the required power. The first part of flows $\lambda_{1}, \lambda_{2}$ and $\lambda_{3}$ from inputs 1,2 and 3 , respectively are reflected by their semi-reflecting mirrors and are summed in input 1 port in the form of $\lambda_{1}+\lambda_{2}+\lambda_{3}$. The second part of flows $\lambda_{1}, \lambda_{2}$ from inputs 1 and 2 passing through the first semi-reflecting mirror and being reflected by the second semi-reflecting mirror respectively is summed in input 2 in the form of $\lambda_{1}+\lambda_{2}$. The flow $\lambda_{3}$ from input 3 passing through the first one and being reflected by the third semi-reflecting mirror is transmitted to input 3 in the form of $\lambda_{3}$.

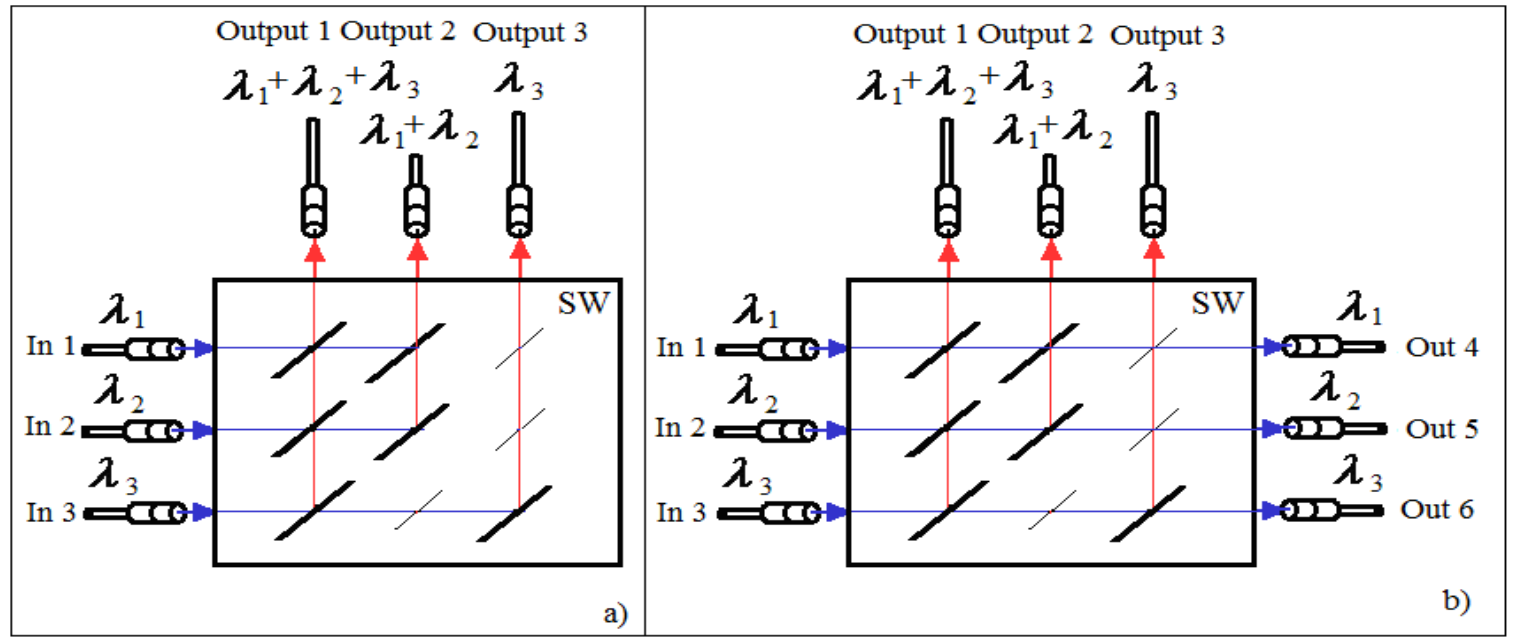

Figure 7: Commutating input optical signals 
In the sample presented in fig.7, b all light beams are likewise divided into flows with the required power. Moreover, flows $\lambda_{1}, \lambda_{2}$ and $\lambda_{3}$ from inputs 1,2 and 3, a similar path takes place according to fig. $7, \mathrm{a}$ and in addition, in contrast to fig. 7 , a all flows $\lambda_{1}, \lambda_{2} \lambda_{3}$ simultaneously are transferred accordingly to outputs 4,5 and 6 in the form of $\lambda_{1}, \lambda_{2} \lambda_{3}$.

The sample given in fig. 8 shows an optical commutator where all the beams can simultaneously pass mutually-opposite to different wavelengths, with different combinations, and they are previously divided into flows with the required power. As it is seen from fig. 8 all ports are both incoming and outgoing.

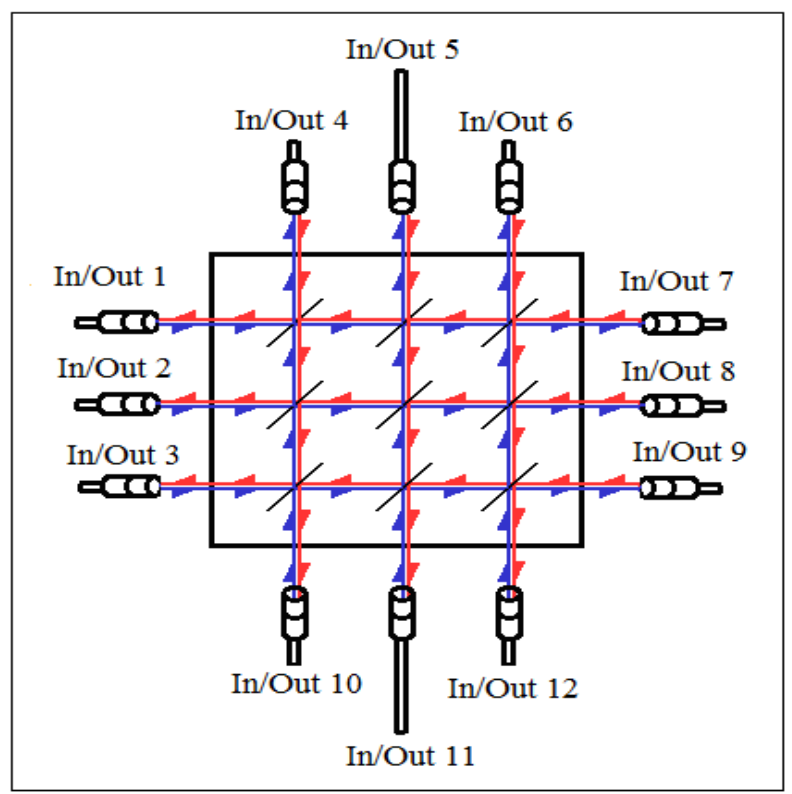

Figure 8: Multifunctional commutator

\section{Conclusion and Recommendations}

Taking into account the principle of optical commutator operation described in figures 6 and 7 , we can conclude that, it is possible to create a multifunctional full optical commutator (fig. 8) on the base of the proposed diagram.

Thus, the original principle of constructing a multi-channel and multifunctional optical commutator is proposed and it is shown that the proposed principle expands the functionality of an optical network.

\section{References}

[1] G.S.Gayvorovskaya, A.V.Ryabsov. Features of using optical commutators in modern information networks. Applicable Information Models . Sofia, ITHEA, 2011, N22, pp.169-181.

[2] V.S.Berikashvili, V.I.Nefedov, D.R.Barski and others. Multi-channel fiber optic telecommunication networks and systems. Proceedings of the International Scientific and 
Technical Conference, November 21-25, 2016. Moscow, MIREA, INTERMATIC-2016, part 5, p.7-14.

[3] United States N. 7.301.177 B2 http://www.uspto.gov.

[4] United States N. 6.445.841 B1 http://www.uspto.gov.

[5] Maharramov V.A. Infra-red observations technique of space objects (Fundamentals of theory and calculation), Baku, Elm, 1999, 336 p.

[6] A.A.Bobsov, V.I.Boikov, S.V.Bistrov, V.V.Grigoriev. Implementing devices and systems for micromovements. - SPB GUITMO, 2011, 131 p.

[7] M.G.Hasanov. Efficiency of a piezoelectric deflector for a light beam in communication technology. AzSTU, №2 (66), Baku, 2010, pp.44-46.

[8] M.H.Hasanov, V.A.Maharramov. Application of piezoelectric light beam deflector in optical commutators of fiber-optical communication networks. The international science-technical jornal HEARD of the Azerbaijan Engineering Academy. Vol. 9, N. 2, 2017, pp. 116-121.

[9] M.G.Hasanov, V.S.Vishnevski. Piezoelectric reversible drive. USSR № 1827708, 1995.

[10] A.V.Ryabsov. Piezo-optic scanning commutators. Information Theories and Applications, Vol.20.Number 3, 2013, pp.295-299.

[11] A.M.Leonov. Piezoceramic light deflector. Optical journal. Volume 77, N.10, 2010, pp.61-64.

[12] A.Samarin. Miniature linear piezoelectric motors. Components and technologies. Faynstrit publishing house, St. Petersburg, 2006, N. 63, p. 36-41.

[13] D.V.Shtikh. Linear displacements with micron and submicron accuracy. Samara Scientific Center of the Russian Academy of Sciences. Volume 14, №1 (2), 2012, pp. 699-652.

[14] T.K.Ismailov, V.A.Maharramov. Scanning device. N 1283698 A2 of the USSR, B.I, 1987, N2, MKI G02 B26/10.

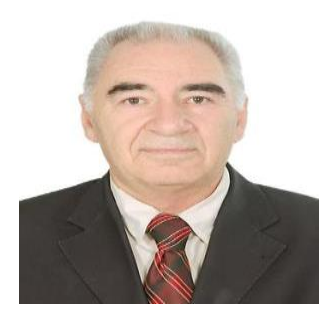

\section{Information about the author:}

Head of the department "Radio engineering and television systems" of the Azerbaijan Technical University, Doctor of Physical and Mathematical Sciences, Professor Vagif Maharramov.

Number of scientific publications: 120

Field of interest: Radio engineering, television, astrophysics.

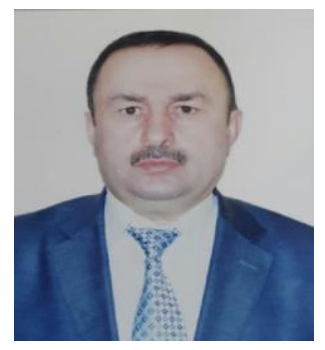

\section{Information about the author:}

Head of the department "Multichannel telecommunication systems" of the Azerbaijan Technical University. Associate Professor Mehman Hasanov Number of scientific publications: 65

Field of interest: Analysis of optical telecommunication systems. 